\title{
Yin Yang 1 is required for PHD finger protein 20-mediated myogenic differentiation in vitro and in vivo
}

\author{
Hyunji Lee ${ }^{1,2} \cdot$ Youngeun Hong ${ }^{1,2} \cdot$ Gyeyeong Kong ${ }^{1,2} \cdot$ Dong Hoon Lee ${ }^{3}$ Minhee Kim ${ }^{1,2} \cdot$ Quangdon Tran ${ }^{1,2}$. \\ Hyeonjeong $\mathrm{Cho}^{1,2} \cdot$ Chaeyeong Kim ${ }^{1,2}$. Sungjin Park ${ }^{1,2} \cdot$ Seon-Hwan Kim ${ }^{4} \cdot$ So Hee Kwon $\mathbb{1}^{3} \cdot$ Jongsun Park $\mathbb{D}^{1,2}$. \\ Jisoo Park ${ }^{1,2,5}$
}

Received: 29 September 2019 / Revised: 9 June 2020 / Accepted: 10 June 2020 / Published online: 18 June 2020

(c) The Author(s), under exclusive licence to ADMC Associazione Differenziamento e Morte Cellulare 2020

\begin{abstract}
The development of skeletal muscle requires progression of a highly ordered cascade of events comprising myogenic lineage commitment, myoblast proliferation, and terminal differentiation. The process of myogenesis is controlled by several myogenic transcription factors that act as terminal effectors of signaling cascades and produce appropriate developmental stage-specific transcripts. PHD finger protein 20 (PHF20) is a multidomain protein and subunit of a lysine acetyltransferase complex that acetylates histone $\mathrm{H} 4$ and p53, but its function is unclear. Notably, it has been reported that PHF20 knockout mice die shortly after birth and display a wide variety of phenotypes within the skeletal and hematopoietic systems. Therefore, the putative role of PHF20 in myogenic differentiation was further investigated. In the present study, we found that protein and mRNA expression levels of PHF20 were decreased during myogenic differentiation in $\mathrm{C}_{2} \mathrm{C}_{12}$ cells. At the same time, Yin Yang 1 (YY1) was also decreased during myogenic differentiation. PHF20 overexpression increased YY1 expression during myogenic differentiation, together with a delay in MyoD expression. PHF20 expression enhanced the transcriptional activity of YY1 while shRNA-mediated depletion of PHF20 resulted in the reduction of YY1 promoter activity in $\mathrm{C}_{2} \mathrm{C}_{12}$ cells. In addition, PHF20 directly bounds to the $\mathrm{YY} 1$ promoter in $\mathrm{C}_{2} \mathrm{C}_{12}$ cells. In a similar manner, $\mathrm{YY} 1$ expression was elevated while myosin heavy chain expression was decreased in PHF20 transgenic (TG) mice. Histological analysis revealed abnormalities in the shape and length of muscles in PHF20-TG mice. Furthermore, PHF20-TG muscles slowly regenerated after cardiotoxin injection, indicating that PHF20 affected muscle differentiation and regeneration after injury in vivo. Taken together, these results suggested that PHF20 plays an important role in myogenic differentiation by regulating YY1.
\end{abstract}

Edited by R. Kitsis

Supplementary information The online version of this article (https:// doi.org/10.1038/s41418-020-0580-6) contains supplementary material, which is available to authorized users.

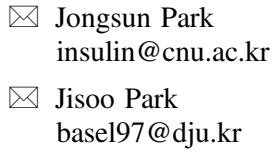

1 Department of Pharmacology, College of Medicine, Chungnam National University, Daejeon 35015, South Korea

2 Department of Medical Science, Metabolic Syndrome and Cell Signaling Laboratory, Institute for Cancer Research, College of

\section{Introduction}

PHD finger protein 20 (PHF20) is a multidomain protein and subunit of a lysine acetyltransferase complex that acetylates histone H4 [1]. PHF20 has been shown to be a transcription factor, and was initially identified in glioma patients [2]. PHF20 knockout (KO) mice die shortly after

Medicine, Chungnam National University, Daejeon 35015, South Korea

3 College of Pharmacy, Yonsei Institute of Pharmaceutical Sciences, Yonsei University, Incheon 21983, South Korea

4 Department of Neurosurgery, Institute for Cancer Research, College of Medicine, Chungnam National University, Daejeon 35015, South Korea

5 Department of Life Science, Hyehwa Liberal Arts College, Daejeon University, Daejeon 34520, South Korea 
birth and display a wide variety of phenotypes within the skeletal and hematopoietic systems [3]. However, the detailed mechanism of these phenotypes in PHF20-KO mice has not been reported.

Skeletal muscle mass represents $40-50 \%$ of the human body weight, making it the largest tissue mass in the body [4]. The development of skeletal muscle requires progression of a highly ordered cascade of events comprising myogenic lineage commitment, myoblast proliferation, and terminal differentiation [5]. The process of myogenesis is controlled by several myogenic transcription factors that act as terminal effectors of signaling cascades, and produces appropriate developmental stage-specific transcripts [6]. The differentiation stage is controlled by a complex network of musclespecific transcription factors (TFs) including the myogenic differentiation marker (MyoD) family, the myocyte enhancer factor-2 (MEF2) family (MEF2A-D), and other general TFs [7]. Yin Yang 1 (YY1) binds DNA through four C-terminal zinc finger domains and can function as an activator or repressor of gene expression [8]. In skeletal muscle, the treatment of rapamycin, inhibitor of mammalian target of rapamycin (mTOR), decreased the gene expression of the mitochondrial transcriptional regulators, including peroxisome proliferator-activated receptor $\gamma$ coactivator $1 \alpha$ (PGC$1 \alpha$ ), estrogen-related receptor $\alpha$, and nuclear respiratory factors, resulting in a decrease in mitochondrial gene expression and oxygen consumption [9]. mTOR phosphorylation levels and its downstream targets, YY1 and PGC- $1 \alpha$, are increased by fibroblast growth factor 21 (FGF21) treatment in $\mathrm{C}_{2} \mathrm{C}_{12}$ myoblasts. Activation of the mTOR-YY1-PGC- $1 \alpha$ pathway by FGF21 in myoblasts regulates energy homeostasis as shown by significant increases in intracellular ATP synthesis, oxygen consumption rate, activity of citrate synthase, glycolysis, mitochondrial DNA copy number, and induction of the expression of key energy metabolic genes [7, 10]. In the present study, the functional roles of PHF20 in skeletal muscle differentiation and developing skeletal muscle in vivo were determined. Notably, PHF20 prevented myogenic differentiation of $\mathrm{C}_{2} \mathrm{C}_{12}$ cells and inhibited the formation of myotubes from myoblasts, while its knockdown accelerated myogenic differentiation. PHF20-mediated enhancement of YY1 transcription was also observed in $\mathrm{C}_{2} \mathrm{C}_{12}$ cells using luciferase-driven promoter assays and chromatin immunoprecipitation (ChIP). Furthermore, PHF20 overexpression resulted in round-shaped muscle fibers and a decreased number of fibers in PHF20 transgenic (TG) mice, when compared with wild-type (WT) control mice. In addition, reduced expression of myosin heavy chain (MHC) was observed in muscle of PHF20-TG mice. Interestingly, overexpression of PHF20 inhibits muscle differentiation as well as regeneration after injury in vitro and in vivo. Taken together, these results suggested a mechanism involving PHF20mediated YY1 regulation, which could explain how muscle function is affected in PHF20-TG mice, and may provide novel therapeutic targets for the treatment of muscle degenerative diseases.

\section{Materials and methods}

\section{Antibodies and reagents}

Anti-PHF20 antibody was purchased from Cell Signaling Technology (Beverly, MA). Anti- $\beta$-actin antibody was obtained from Sigma-Aldrich (St. Louis, USA). Anti-YY1, anti-MyoD, and anti-MHC antibodies were from Santa Cruz Biotechnology (Santa Cruz, CA). Anti-myosin (MF20) antibodies were from DSHB, University of Iowa. HRPconjugated anti-mouse, anti-rabbit, and anti-goat IgG antibodies were obtained from Koma biotech (Seoul, Korea). pYY-luciferase was purchased from Panomics (California, USA). Luciferase assay system kit was purchased from Promega (Madison, USA). Tet-on advanced inducible gene expression system kit was obtained from Clontech (Takara Bio Company, USA).

\section{Cell culture}

$\mathrm{C}_{2} \mathrm{C}_{12}$ cells were maintained in DMEM (WELGENE, Gyeongsan, Korea) supplemented with $10 \%$ fetal bovine serum (HyClone, Logan, USA) and 1\% Anti-anti (Gibco, Detroit, USA) at $37^{\circ} \mathrm{C}$ in $5 \% \mathrm{CO}_{2}$. Differentiation was initiated $24 \mathrm{~h}$ after seeding by changing to differentiation medium (DM) consisting of DMEM supplemented with 2\% horse serum and 1\% Anti-anti (Gibco, Detroit, USA). The medium was changed for every 2 days.

\section{Establishment of $\mathrm{C}_{2} \mathrm{C}_{12} /$ Tet-On-inducible PHF20 cells}

$\mathrm{C}_{2} \mathrm{C}_{12}$ cells were treated with lentivirus-TET3G and selected with $\mathrm{G} 418(1.2 \mathrm{mg} / \mathrm{ml})$ to generate $\mathrm{C}_{2} \mathrm{C}_{12}$-TET3G myoblast cells. Next, $\mathrm{C}_{2} \mathrm{C}_{12}$-TET3G cells were transiently transfected with pLVX-TRE3G-PHF20 and selected with puromycin $(3 \mu \mathrm{g} / \mathrm{ml})$ for Tet-On inducible PHF20 cells. The expression of PHF20 was confirmed in the media with doxycycline (Doxy).

\section{Immunoblot analysis}

Western blotting was performed as previously described $[11,12]$. After the completion of experimental conditions, cells were placed on ice and extracted with lysis buffer containing $50 \mathrm{mM}$ Tris-HCl, pH7.5, $1 \%$ v/v Nonidet P-40, $120 \mathrm{mM} \mathrm{NaCl}, 25 \mathrm{mM}$ sodium fluoride, $40 \mathrm{mM} \beta$-glycerol phosphate, $0.1 \mathrm{mM}$ sodium orthovanadate, $1 \mathrm{mM}$ phenylmethylsulfonyl fluoride, $1 \mathrm{mM}$ benzamidine, and $2 \mu \mathrm{M}$ 
microcystin-LR. Lysates were centrifuged for $30 \mathrm{~min}$ at $13,000 \mathrm{rpm}$. The cell extracts were resolved by $10.0-7.5 \%$ SDS-PAGE, and transferred to Immobilon-P membranes (Millipore). The filters were blocked for $1 \mathrm{~h}$ in $1 \times$ tribuffered saline buffer $(140 \mathrm{mM} \mathrm{NaCl}, 2.7 \mathrm{mM} \mathrm{KCl}$, and $250 \mathrm{mM}$ Tris-HCl, pH7.4), containing 5\% skimmed milk and $0.2 \%$ Tween-20, followed by an overnight incubation with each protein's primary antibodies diluted 1000 folds at $4{ }^{\circ} \mathrm{C}$. The secondary antibody was horseradish peroxidaseconjugated anti-mouse IgG or anti-rabbit IgG (Koma biotech, Seoul, Korea), diluted 2000-fold in the blocking buffer. The detection of protein expression was visualized by enhanced chemiluminescence, according to the manufacturer's instructions.

\section{Real-time quantitative reverse transcription- polymerase chain reaction (qRT-PCR)}

$\mathrm{C}_{2} \mathrm{C}_{12}$ /Tet-On inducible PHF20 cells were plated in 6-well plate. After $24 \mathrm{~h}$, doxycycline was treated to induce the PHF20 expression. The following day, total RNA was extracted. The cDNA was synthesized from total RNA. The qRT-PCR measurement of individual cDNAs was performed using SYBR green dye to measure duplex DNA formation with the StepOne Plus real-time PCR system (Invitrogen, Carlsbad, CA). qRT-PCR analysis conducted by isolating RNA using Total RNA was isolated using the QuickGene RNA kit (Fujifilm's Life Science System, Tokyo, Japan). Complementary DNA was produced with the SuPrimeScript RT Premix (GeNet Bio, Cheonan, Korea). The reactions were up in triplicate with the Prime Q-Mastermix (GeNet Bio, Cheonan, Korea) and run on CFX96T Real-time System (Bio-Rad, Hercules, CA, USA). The sequences of the primers that were used for the qPCR were as follows; mouse GAPDH, forward (5'-TCCAG TATGACTCCACTC- $3^{\prime}$ ), reverse (5'-ATTTCT CGTGGTT CACAC-3'); mouse PHF20, forward (5'-CATTGACTAC GAAGAAGGGAG-3'), reverse (5'-CTTCTCTAAAGGG CGCAGATA-3'); mouse YY1, forward (5'-CAGAAGC AGGTGCAGATC AGACCCT-3'), reverse (5'-GCACCAC CACCCACGGAATCG-3'); mouse MyoD, forward (5'-TA CAGTGGCGACTCAGATGC-3') and reverse (5'-GAGA TGCGC TCCACTATGCT-3').

\section{Detection of MHC by immunofluorescence}

$\mathrm{C}_{2} \mathrm{C}_{12}$ /Tet-On-inducible PHF20 cells were plated at a density of $1 \times 10^{5}$ cells/well in 12 -well plates containing sterile coverslips and grown at $37^{\circ} \mathrm{C}$. Cells were treated with doxycycline or not for $24 \mathrm{~h}$. After $24 \mathrm{~h}$ cells were differentiated by changing the DM until 1 and 3 days. And premyocytes were not changed DM. Next the cells were washed twice with PBS prewarmed to $37^{\circ} \mathrm{C}$ and fixed to the coverslips by incubating in $4 \%$ paraformaldehyde for $15 \mathrm{~min}$. Cells were then washed twice with PBS. Before blocking, cells were incubated PBS with Triton $\mathrm{x}-100$ for $15 \mathrm{~min}$. The coverslips were blocked in $1 \%$ BSA for $1 \mathrm{~h}$ at room temperature with shaking. Anti-myosin (MF20) antibody was added (1:200) in 1\% BSA and incubated for overnight with shaking at $4{ }^{\circ} \mathrm{C}$. Coverslips were then washed three times each with PBS. FITC secondary antibody (1:1000) in 5\% skim milk was then added and incubated for $6 \mathrm{~h}$ with shaking at room temperature in the dark. Coverslips were washed three times with PBS and mounted onto slides using mounting medium with DAPI VECTASHIELD (St. Louis, USA). For immunofluorescence analysis of muscle tissues, paraffin sections were deparaffinized with xylene and rehydrated in a graded series of ethanol. The slide was submerged in peroxidase quenching solution for $10 \mathrm{~min}$. After washed twice with PBS for $5 \mathrm{~min}$, it was added with 2 drops of blocking solution and incubated for $30 \mathrm{~min}$. Following washed twice with PBS, the primary antibody (anti-MHC) was applied at $4{ }^{\circ} \mathrm{C}$ for overnight. FITC secondary antibody (1:1000) in blocking solution were then added and incubated for $1 \mathrm{~h}$ at room temperature in the dark. Coverslips were washed three times with PBS and mounted onto slides using mounting medium with DAPI VECTASHIELD.

\section{Luciferase reporter gene assay}

$\mathrm{C}_{2} \mathrm{C}_{12} /$ Tet-On-inducible PHF20 cells $\left(3 \times 10^{5}\right.$ cells $\left./ \mathrm{ml}\right)$ were transfected with $1 \mu \mathrm{g}$ of plasmids containing YY1-luciferase in 6-well plate according to the manufacturer's protocol. The cells were used for experiments $24 \mathrm{~h}$. Cells were lysed in reporter lysis buffer containing $25 \mathrm{mM}$ Tris-phosphate, $2 \mathrm{mM}$ DTT, $2 \mathrm{mM}$ trans-1,2-cyclohexane diamine-N,N,N', $\mathrm{N}^{\prime}$-tetraacetic acid, $10 \%(\mathrm{v} / \mathrm{v})$ glycerol, and $1 \%(\mathrm{v} / \mathrm{v})$ Triton $\mathrm{x}-100$. Luciferase activity was determined in a luminometer (Duo Lumat LB 9507) by incubating $20 \mu \mathrm{g}$ of cell lysate with luciferase assay substrate (Promega) and measuring light emission for $10 \mathrm{~s}$.

\section{Chromatin-immunoprecipitation assays}

ChIP assays were performed following the manufacturer's protocol (Upstate Biotechnology, Lake Placid, NY, USA). $\mathrm{C}_{2} \mathrm{C}_{12}$ cells were differentiated for 3 days and treated with formaldehyde for $7.5 \mathrm{~min}$, were washed two times with PBS containing protease inhibitor, and harvested using SDSlysis buffer $(50 \mathrm{mM}$ Tris- $\mathrm{HCl} \mathrm{pH}$ 8.0, $1 \%$ SDS, $10 \mathrm{mM}$ EDTA, $1 \mathrm{mM}$ PMSF, $1 \mu \mathrm{g} / \mathrm{ml}$ aprotinin, $2 \mu \mathrm{g} / \mathrm{ml}$ pepstatin) for $10 \mathrm{~min}$ on ice. The lysate was incubated with primary antibody or control $\mathrm{IgG}$ for overnight at $4{ }^{\circ} \mathrm{C}$. And then, mixed samples were incubated with $30 \mu$ of Protein A beads containing $500 \mu \mathrm{g} / \mathrm{ml} \mathrm{BSA,} 200 \mu \mathrm{g} / \mathrm{ml}$ salmon sperm 
DNA for $3 \mathrm{~h}$ at $4{ }^{\circ} \mathrm{C}$. DNA samples were purified using a QIA quick PCR purification kit (Qiagen, Hilden, Germany) and quantified by qRT-PCR and the data are expressed as a percentage of input. The promoter-specific primers used were, for mouse YY1 \#1 (-870 -760): $5^{\prime}$ CCTTCACCCCAGCTCG TT-3' (forward), 5'GCGGTGCAACAGTGACAA-3' (reverse), \#2 (-759 -631): 5'-GGCACTTTTG TCACTGTTGCAC-3' (forward), 5'-AACCAGGGTCCTCCCGATT-3', \#3 (-630 -564): 5'-ATCGG GAGGACCCTGGTT-3' (forward), 5'CGATTCCCCACTCGCTGT-3' (reverse), \#4 (-563 -450): 5'-ACAGCGAGTGGGGAATCG-3' (forward), 5'CGCCTCCTTCCACACAAA-3' (reverse), \#5 (-449 -317): 5'-ACAGCGAGTGGGGAATC-3' (forward), 5'CGCCTCCTTCCACACAAA-3' (reverse), \#6 (-316 -201): 5'-TGAGGAGTTGGTATTTGTGTGGAA-3' (forward), 5'-GCTGCCTGTCC TGCTTCC-3' (reverse), \#7 $(-200 \sim-55):$ 5'-CGCTGCCTTCC TCCCTCT-3' (forward), 5'-CGTCCG TGGCGATGTAGA-3' (reverse), \#8 (-54 34): 5'-TC GGGCGACACCCTCT-3' (forward), 5'ATGG TCTCCACCGGGATG-3' (reverse) and \#9 (33 144): 5'-GACCATCCCGGTGGAGAC-3' (forward), 5'GGTGGTGGTGGTGGTGGT-3' (reverse).

\section{PHF20-TG model mice}

The cDNA encoding human PHF20 was subcloned downstream of CMV promoter in pcDNA3 vector to generate TG mice. Positive founds were identified by southern blotting and bread to WT C57BL/6 mice for four generations. PHF20-TG and WT were maintained in a controlled environment (12 h light/12 h dark cycle; humidity 50-60\%; ambient temperature $22{ }^{\circ} \mathrm{C}$ ). All mouse experiments were performed in the animal facility according to institutional guidelines. The experimental protocols were approved by the institutional review board of Chungnam National University (CNU-00890).

\section{Muscle regeneration assay}

Cardiotoxin (CTX, Sigma-Aldrich, USA) injury and muscle regeneration assay followed as described by Glynnis et al. [13]. Briefly, $30 \mu \mathrm{L}$ of CTX $(10 \mu \mathrm{M})$ was injected into the tibialis anterior (TA) muscles of 3-month-old WT and PHF20-TG mice. TA muscles harvested at 0,5 , and 10 days post injection were fixed in $4 \%$ paraformaldehyde.

\section{Isolation and culture of mouse primary myoblasts}

TA muscles were carefully isolated from 3-month-old mice and digested with collagenase I (Sigma-Aldrich, USA). The muscles were dissected and incubated in dissociation buffer containing $0.2 \%$ collagenase $\mathrm{I}$ in serum-free DEME at
$37{ }^{\circ} \mathrm{C}$ for $60 \mathrm{~min}$. The digested muscle was placed in a $6-\mathrm{cm}$ Petri dish precoated with horse serum, and myoblasts were isolated and transferred onto matrigel (Coning, USA)coated culture dishes. Myoblasts from WT or PHF20-TG mouse donors were cultured for the same number of passages and used in all experiments. To induce differentiation of primary myoblasts, cells were grown to $90 \%$ confluence in growth medium and incubated in DM for 7 days.

\section{Satellite cell isolation and culture}

Skeletal muscle forms the hindlimbs of 3-month-old WT, and PHF20-TG mice were isolated and carefully discard pieces of fat. The tissue was digested with PBS containing $500 \mathrm{U} / \mathrm{ml}$ collagenase type II (Sigma-Aldrich, USA), $1.5 \mathrm{U} /$ $\mathrm{ml}$ collagenase D (Sigma-Aldrich, USA), $2.5 \mathrm{U} / \mathrm{ml}$ dispase II (Roche, USA), and $2.5 \mathrm{mM} \mathrm{CaCl} 2$ for $60 \mathrm{~min}$ in $37^{\circ} \mathrm{C}$ water bath. The Satellite cells were cultured in DMEM with $20 \%$ fetal bovine serum, $10 \mathrm{ng} / \mathrm{ml}$ basic fibroblast growth factor, and $1 \%$ Anti-anti on $10 \%$ matrigel-coated cell culture plates at $37^{\circ} \mathrm{C}$ in a $5 \% \mathrm{CO}_{2}$. To induce differentiation, cells at $70 \%$ confluency were changed to DM (5\% horse serum and $1 \%$ Anti-anti) for 3 or 10 days.

\section{Histological analysis and immunohistochemistry}

Muscles were collected and fixed in $4 \%$ paraformaldehyde overnight at $4{ }^{\circ} \mathrm{C}$. Fixed samples were embedded in paraffin and $5 \mu \mathrm{m}$ sections were cut and mounted on slides to generate a series of six slides with comparable adjacent sections. For general morphological analysis, one slide of each series was hematoxylin and eosin (H\&E)-stained as previously described [8]. For immunohistological analysis, paraffin sections were deparaffinized with xylene and rehydrated in a graded series of ethanol. The slide was submerged in peroxidase quenching solution for $10 \mathrm{~min}$. After washed twice with PBS for 5 min, it was added with two drops of Reagent A for blocking and incubated for 30 min. Following washed twice with PBS, the primary antibody (anti-MHC) was applied at $4{ }^{\circ} \mathrm{C}$ for overnight. Then, biotinylated secondary antibody, Reagent B, was added after rinsed with PBS. It was incubated at room temperature for $1 \mathrm{~h}$. It was rinsed with PBS and dropped with enzyme-conjugated Reagent C. After washed with PBS, DAB chromogen, mixture of Reagent D1, D2, and D3, was dropped and observed signals with florescence microscope. Then, it was stopped with distilled water and took pictures with florescence microscope.

\section{Statistical analysis}

GraphPad instat software (GraphPad Prism) was used for statistical analyses. Data are expressed as the mean 
A

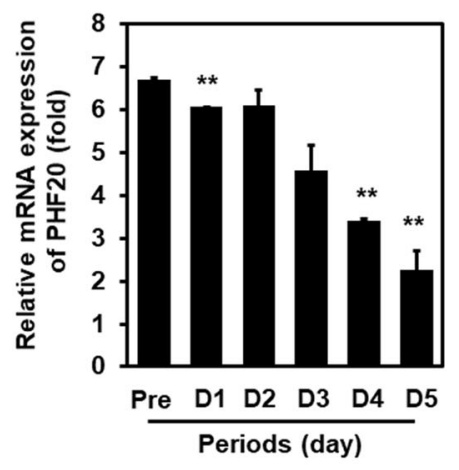

D

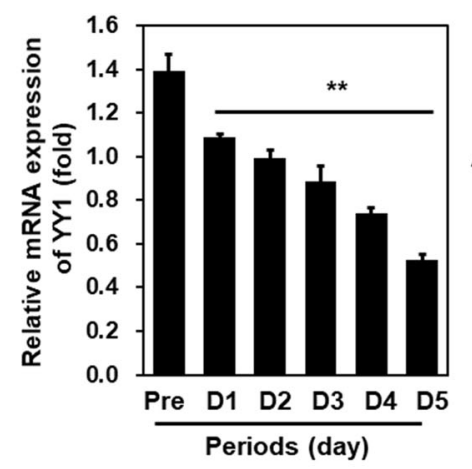

B

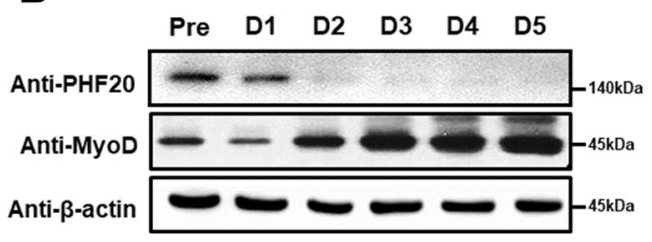

C PHF20

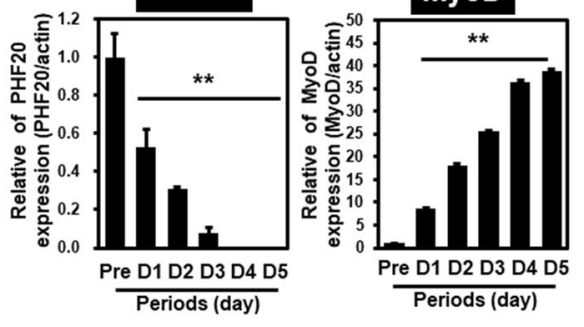

$\mathbf{E}$

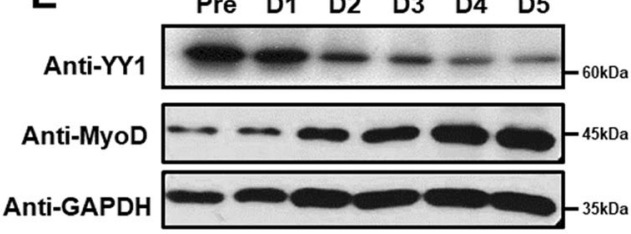

$\mathbf{F}$

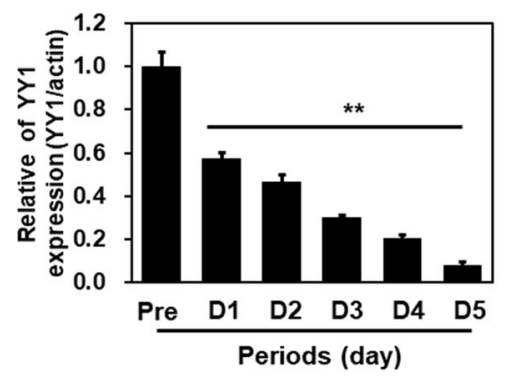

of the corresponding immunoblot data. Relative and statistical differences in PHF20 and MyoD expression were determined by normalizing values for actin in each lane, with a set value for premyocytes as 1. d Changes in mRNA levels of YY1 were analyzed using quantitative real-time PCR. e Changes in protein levels were analyzed by western blotting with the corresponding antibodies (YY1, MyoD, and $\beta$-actin). Results are representative of three independent experiments. f Relative and statistical differences of YY1 were plotted during myogenic differentiation. All data are expressed as the mean \pm SEM of three independent experiments $(* * p<0.01)$, using one-way ANOVA with Tukey's correction for multiple comparisons.

\section{Results}

\section{Reduction of PHF20 expression in myogenic differentiation together with YY1 expression}

It has been reported that PHF20-KO mice display various phenotypes, including defects in skeletal and hematopoietic systems [3]. In the present study, the possible involvement (version 1.47) 

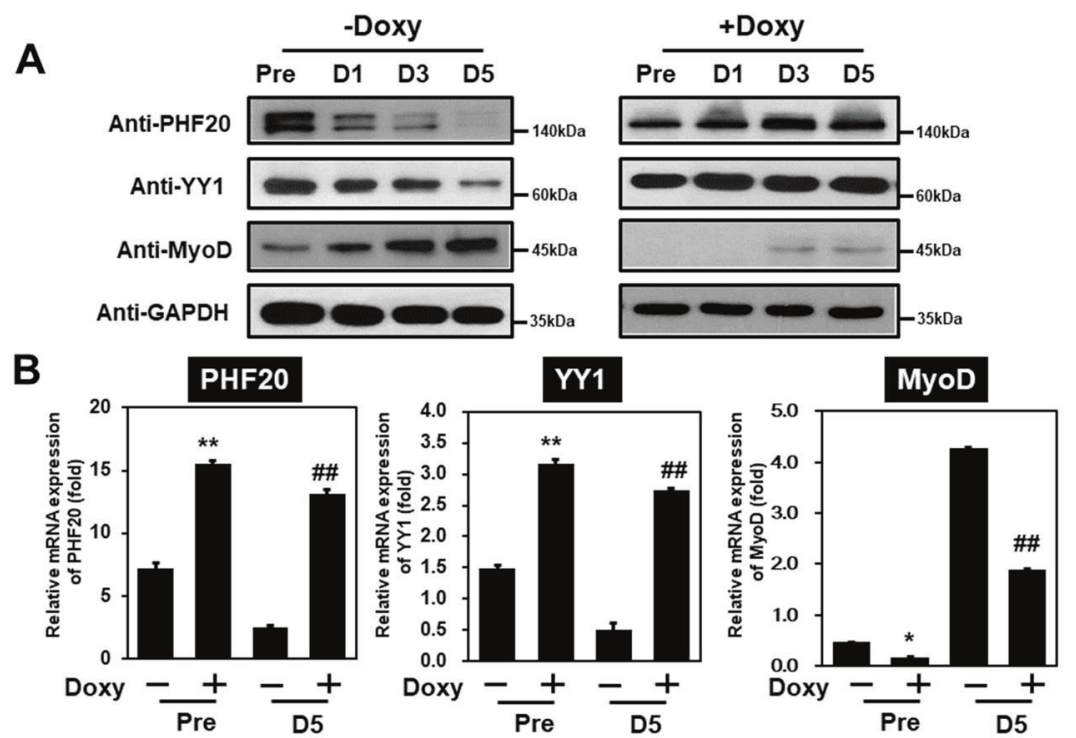

C
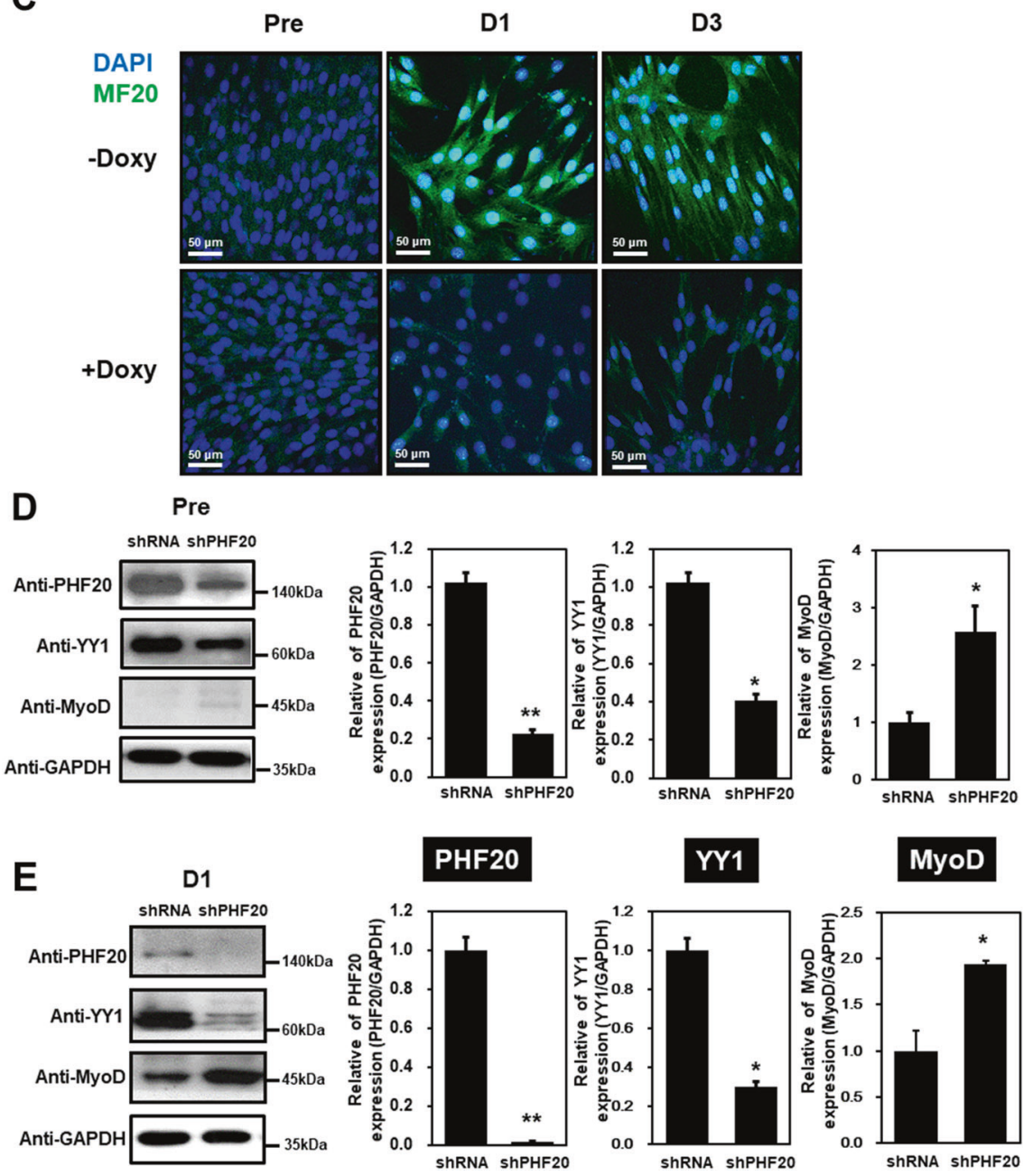

of PHF20 in myogenic differentiation was investigated. As a first attempt in evaluating the changes of PHF20 in myogenic differentiation, the mRNA and protein levels of
PHF20 were monitored by RT-qPCR and western blot analyses. Notably, the levels of PHF20 (mRNA, Fig. 1a; protein, Fig. 1b, c) were gradually decreased during 
Fig. 2 Effect of PHF20 on myogenic differentiation in $\mathrm{C}_{2} \mathrm{C}_{12}$ cells. a Immunoblots were prepared from $\mathrm{C}_{2} \mathrm{C}_{12}$ myoblasts (Pre) and differentiated myotubes (D1, D3, and D5), either untreated (-Doxy) or treated (+Doxy), with doxycycline for $24 \mathrm{~h}$, and cell lysates were prepared from these cells. Expression levels of PHF20, YY1, and MyoD were determined using western blot analysis with corresponding antibodies. Results are representative of three independent experiments. b Total RNA from $\mathrm{C}_{2} \mathrm{C}_{12}$ myoblasts (Pre) and differentiated myotubes (D5), either untreated (-Doxy) or treated ( + Doxy), with doxycycline was analyzed by real-time qPCR of PHF20, YY1, and MyoD. Results are expressed as the mean \pm SEM of three independent experiments $(* p<0.05 ; * * p<0.01$, pre -Doxy versus pre +Doxy samples; ${ }^{\# \#} p<0.01$, D5 -Doxy versus D5 +Doxy samples), using one-way ANOVA with Tukey's correction for multiple comparisons. $\mathrm{C}_{2} \mathrm{C}_{12}$ myoblasts (Pre) and differentiated myotubes (D1 and D3), either untreated (-Doxy) or treated (+Doxy), with doxycycline were prepared. Cells were further processed by immunofluorescence using anti-MF20 antibody (green). Nuclei were counterstained with 4',6-diamidino-2-phenylindole (DAPI; blue). The sample was analyzed by confocal microscopy. Scale bars, $50 \mu \mathrm{m}$. d Stable $\mathrm{C}_{2} \mathrm{C}_{12}$ myoblast cells (Pre) were constructed using a lentivirus expressing shRNA for scrambled sequences (shRNA) or PHF20 sequence (shPHF20). After $24 \mathrm{~h}$, cell lysates were analyzed by immunoblotting with the corresponding antibodies. Results are representative of three independent experiments. Data are expressed as the mean $\pm \operatorname{SEM}(* p<0.05 ; * * p<0.01)$, using Student's $t$ test for paired samples. e $\mathrm{C}_{2} \mathrm{C}_{12}$ cells were differentiated (D1) with induced medium and infected with lentivirus shRNA (shRNA) and lentivirus PHF20 (shPHF20) during day 1 . After $24 \mathrm{~h}$, cell lysates were analyzed. Results are representative of three independent experiments. Data are expressed as the mean \pm SEM $(* p<0.05 ; * * p<0.01)$, using Student's $t$ test for paired samples.

myogenic differentiation, when induced by DM containing $2 \%$ horse serum, while protein levels of MyoD were gradually increased (Fig. 1b, c) in $\mathrm{C}_{2} \mathrm{C}_{12}$ cells, suggesting that PHF20 might be involved in myogenic differentiation. PHF20 has been shown to be a transcription factor $[3,8]$. Based on the screening results of transcription factor profiling [14], YY1 was one of the upregulated transcription factors in cells overexpressing PHF20. The changes in YY1 mRNA and protein expression were therefore also monitored, because YY1 has been shown to be involved in myogenic differentiation $[15,16]$. As expected, expression levels of YY1 mRNA and protein were significantly reduced every day during myogenic differentiation (Fig. 1d-f), indicating that expression of PHF20 and YY1 were negatively correlated during myogenic differentiation.

\section{Ectopic expression of PHF20 results in the inhibition of myogenic differentiation in $\mathrm{C} 2 \mathrm{C} 12$ cells}

To further evaluate the effects of PHF20 on myogenic differentiation, $\mathrm{C}_{2} \mathrm{C}_{12} /$ Tet-On-inducible PHF20 cells were prepared. Figure 2a shows that PHF20 and YY1 expression levels were decreased in control cells (Fig. 2a, left panel) during myogenic differentiation, whereas doxycyclineinduced PHF20 expression led to consistent YY1 expression and a delay of $\mathrm{MyoD}$ expression in $\mathrm{C}_{2} \mathrm{C}_{12}$ cells
(Fig. 2a, right panel). Similar results were also observed for expression levels of YY1 and MyoD mRNA (Fig. 2b) during myogenic differentiation (premyoblast and differentiation day 5). To investigate the effects of PHF20 on myogenic terminal differentiation (myotube formation), expression of MHC was monitored using MF20 antibody. Control $\mathrm{C}_{2} \mathrm{C}_{12}$ cells differentiated to myotubes (judged by the morphology of multinucleated myotubes) when they were shifted to DM for 3 days (Fig. 2c, top panel) while doxycycline-induced PHF20 expression resulted in significant inhibition of myotube formation during myogenic differentiation (Fig. 2c, bottom panel). In addition, shRNAmediated knockdown of PHF20 clearly induced the reduction of YY1 expression and the elevation of MyoD expression in premyocytes (Fig. 2d). These effects were more pronounced at differentiation day 1 (Fig. 2e). Taken together, these results suggested that PHF20 played a role in myogenic differentiation.

\section{Transcriptional activity of YY1 is regulated by PHF20 via direct DNA-binding}

Based on the concomitant changes of PHF20 and YY1 expression levels during myogenic differentiation (Figs. 1 and 2), the possible PHF20-mediated regulation of YY1 transcription was further evaluated using a YY1 promoter assay in $\mathrm{C}_{2} \mathrm{C}_{12}$ cells. As expected, doxycyclineinduced PHF20 expression promoted an increase in YY1 promoter activity in a dose-dependent manner (Fig. 3a). Furthermore, shRNA-mediated reduction of PHF20 expression also blocked YY1 promoter activity (Fig. 3b) in undifferentiated $\mathrm{C}_{2} \mathrm{C}_{12}$ cells, suggesting that PHF20 positively regulated $\mathrm{YY} 1$ expression of transcript levels in premyoblasts. To further evaluate the effects of PHF20 on YY1 transcription under physiological conditions, the YY1 promoter assay was conducted using $\mathrm{C}_{2} \mathrm{C}_{12}$ cells during myogenic differentiation. As expected, YY1 promoter activity gradually decreased along with a reduction in PHF20 and YY1 expression, while MyoD expression increased (Fig. 3c). However, doxycycline-induced PHF20 expression led to consistent YY1 promoter activity during myogenic differentiation, while MyoD expression was hardly detected (Fig. 3d), indicating that the degree of PHF20 expression was important in the regulation of myogenic differentiation via YY1. To determine whether PHF20 can directly bind to the YY1 promoter, ChIP assays were employed. As shown in Fig. 3e, PHF20 was significantly bound to the YY1 promoter regions $(-316 /-201$ bp [\#6] and $-200 /-55$ bp [\#7]). However, the binding of PHF20 to YY1 promoter regions was markedly decreased during muscle differentiation in $\mathrm{C}_{2} \mathrm{C}_{12}$ cells. The previous study showed that PHF20 is a core component of the male absent on the first/nonspecific lethal lysine acetyltransferase 

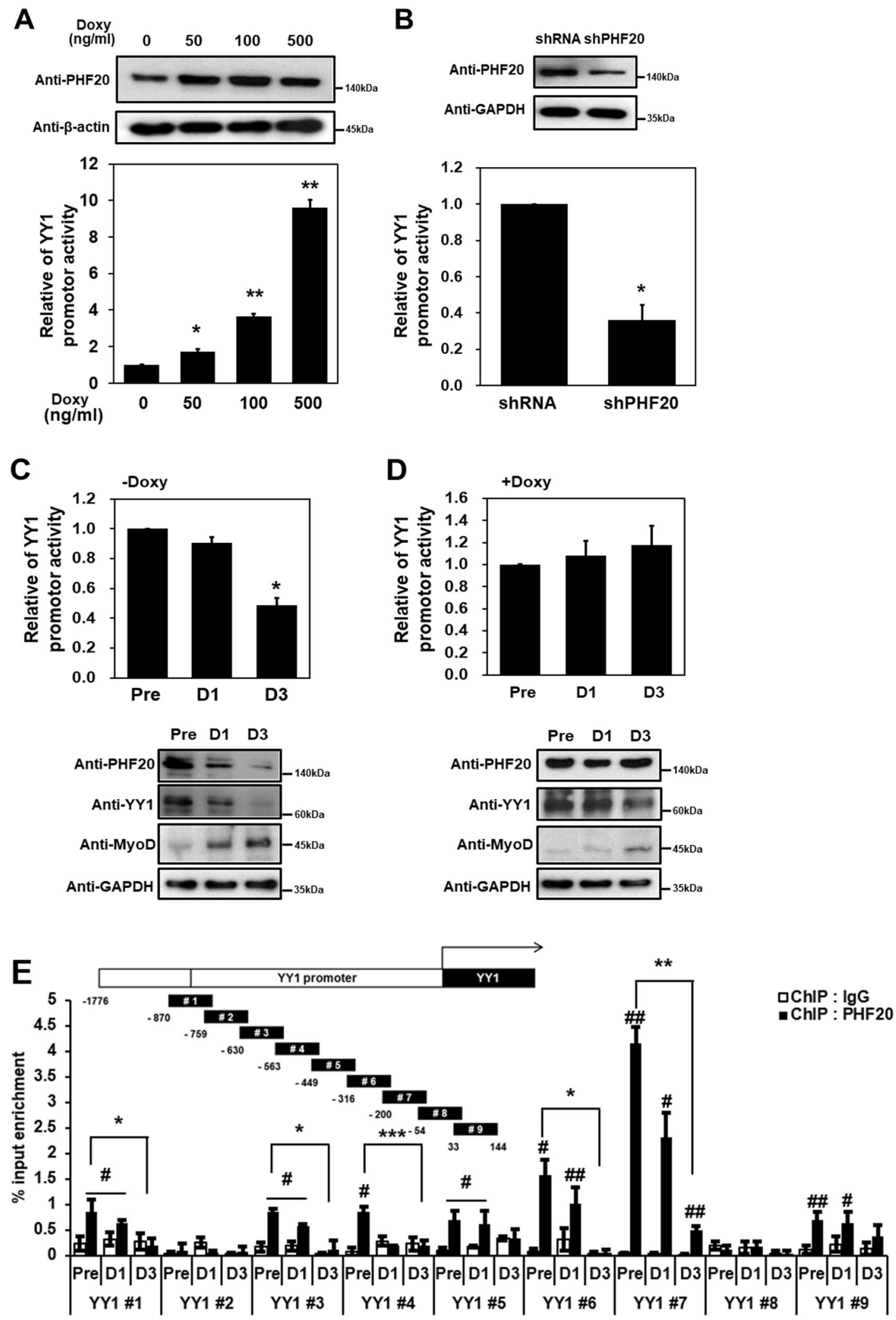

complex responsible for histone $\mathrm{H} 4$ acetylation [17]. Therefore, the changes of histone $\mathrm{H} 4$ acetylation status at the YY1 promoter were monitored. ChIP assay revealed that the levels of $\mathrm{H} 4$ acetylation were substantially reduced on YY1 promoter regions ( $-563 /-450$ bp [\#4], -449/-317 bp [\#5], and $-316 /-201$ bp [\#6]) during muscle differentiation 
Fig. 3 PHF20 directly binds to the promoter of YY1. a $C_{2} C_{12}$ myoblasts were transiently transfected with the YY1-lucreporter gene in growth medium and treated dose-dependently with doxycycline (Doxy). After $24 \mathrm{~h}, \mathrm{C}_{2} \mathrm{C}_{12}$ myoblasts were analyzed for luciferase activity (bottom panel). Before analysis of luciferase activity, $\mathrm{C}_{2} \mathrm{C}_{12}$ myoblasts were confirmed to express PHF20 protein using western blots (top panel). Results are expressed as the mean \pm SEM of three independent experiments $(* p<0.05 ; * * p<0.01)$, using one-way ANOVA with Tukey's correction for multiple comparisons. $\mathbf{b} \mathrm{C}_{2} \mathrm{C}_{12}$ myoblasts were infected with lenti-shRNA (shRNA) or lenti-shPHF20 (shPHF20) and transfected with the YY1-lucreporter gene in growth medium. After $24 \mathrm{~h}$, luciferase activity was determined in $\mathrm{C}_{2} \mathrm{C}_{12}$ myoblasts (bottom panel). Before determination of luciferase activity, $\mathrm{C}_{2} \mathrm{C}_{12}$ myoblasts were confirmed to express PHF20 protein using western blots (top panel). Results are representative of three independent experiments. Data are expressed as the mean \pm SEM $\left({ }^{*} p<\right.$ 0.05), using Student's $t$ test for paired samples. c $\mathrm{C}_{2} \mathrm{C}_{12}$ cells were differentiated with induction medium. At different time points, $\mathrm{C}_{2} \mathrm{C}_{12}$ cells were transfected with the YY1-lucreporter gene without doxycycline. $\mathrm{C}_{2} \mathrm{C}_{12}$ cells were then analyzed for luciferase activity (top panel). Before analysis of luciferase activity, expression of proteins (PHF20, YY1, and MyoD) were analyzed (bottom panel). Results are expressed as the mean \pm SEM of three independent experiments $\left({ }^{*} p<\right.$ 0.05 ), using one-way ANOVA with Tukey's correction for multiple comparisons. $\mathbf{d ~ C}_{2} \mathrm{C}_{12}$ cells were differentiated with induction medium. At different time points, $\mathrm{C}_{2} \mathrm{C}_{12}$ cells were transfected with the YY1lucreporter gene with doxycycline $(100 \mathrm{ng} / \mathrm{mL}) . \mathrm{C}_{2} \mathrm{C}_{12}$ cells were then analyzed for luciferase activity (top panel). Before analysis of luciferase activity, expression of proteins (PHF20, YY1, and MyoD) were analyzed (bottom panel). Results are representative of three independent experiments. Data are expressed as the mean \pm SEM, using oneway ANOVA with Tukey's correction for multiple comparisons. e Chromatin immunoprecipitation (ChIP) analysis of selected regions covering the $\mathrm{YY} 1$ promoter in undifferentiated (Pre) and differentiated (D1 and D3) $\mathrm{C}_{2} \mathrm{C}_{12}$ cells. An IgG was used as a control in the ChIP assays in $\mathrm{C}_{2} \mathrm{C}_{12}$ cells. Error bars represent standard deviation $(n=3)$ $(* * p<0.01 ; * p<0.05$ versus the control samples, Student's $t$ test). Results are representative of three independent experiments.

(Supplementary Fig. S1). Collectively, reducing occupancy of PHF20 correlated with the decreasing levels of $\mathrm{H} 4$ acetylation on the YY1 promoter during muscle differentiation. Taken together, these findings provide further evidence that PHF20 binds directly to the YY1 promoter as a transcription factor to regulate muscle differentiation via modulating YY1 transcription.

\section{PHF20 inhibits MyoD expression via YY1}

It has been reported that YY1 inhibits skeletal muscle differentiation $[7,9,10,18]$. Based on previous findings that PHF20 directly regulates YY1 (Fig. 3e), the effects of PHF20-mediated YY1 function on downstream targets during myogenic differentiation were monitored. Overexpression of PHF20 led to induction of YY1 expression in premyocytes as well as differentiation on day 3 , together with the reduction of MyoD expression (Fig. 4a, the first two lanes for each condition). As previously shown in Fig. 2a, b, this reduction in MyoD levels was recovered by shRNA-mediated depletion of YY1 (Fig. 4a, the last two lanes for each condition). To further evaluate PHF20/YY1mediated myogenic differentiation during physiological events, the formation of multinucleated myotubes was examined using immunofluorescence with anti-MHC antibody. Figure $4 \mathrm{~b}, \mathrm{c}$ shows that when shRNA induced a reduction in YY1 expression, polynuclear myotube formation was further accelerated, while differentiation was inhibited when doxycycline-induced PHF20 expression, indicating that PHF20 indirectly affects MyoD expression through YY1 transcription.

\section{Defects of muscle morphology in PHF20-TG mice}

Because a noticeable phenotype of PHF20-KO mice was reduction of fat storage and weight [3], PHF20-TG mice were prepared from C57BL/6 mice to evaluate these phenotypes in vivo. The characterization of PHF20-TG mice showed that PHF20 expression was significantly increased in skeletal muscle, together with YY1 expression (Fig. 5a, b). However, expression of MHC, a major marker of muscle differentiation, was reduced in PHF20-TG mice (Fig. 5a, b). In order to further evaluate the PHF20 roles in myogenic differentiation in vivo, primary skeletal muscle cells were isolated from WT and PHF20-TG mice. As shown in Fig. 5c, primary cells from WT mice showed that PHF20 expression was reduced after 7 days of differentiation with concomitant changes in YY1 expression. On the contrast, primary cells from PHF20-TG mice displayed high expression of PHF20 and YY1 and low expression of MyoD following 7 days differentiation. In addition, myotube formation was also inhibited in primary cells from PHF20-TG mice after 7 days differentiation (Fig. 5d), confirming that PHF20-mediated suppression of myogenic differentiation was occurred in PHF20-TG mice. Figure 5e shows that gross dissection indicated that muscle color was paler in PHF20-TG mice than in control mice, suggesting that PHF20-TG mice have more fat accumulation than WT mice in muscle. It was assumed that white adipose tissue accumulated where muscles were less developed, making the muscle appear pale. This process was further confirmed by the increase in body weight in PHF20-TG mice, when compared with control mice (Fig. 5f). H\&E staining of muscle tissue revealed that lower numbers of muscle fibers were detected in PHF20-TG mice (Fig. 5g). In addition, the fiber shape of PHF20-TG was mostly round, and large empty spaces between fibers were observed (Fig. $5 \mathrm{~g}$, bottom panel). Longitudinal sections of muscle showed that the integrity of muscles in PHF20-TG mice was less compacted than that in WT control mice (Fig. 5h). A specific length and direction of muscle fibers were found in WT mice, whereas PHF20-TG mice displayed short muscle fiber length and few nuclei. Furthermore, the decrease in MHC expression in the muscles of PHF20-TG mice was 
A

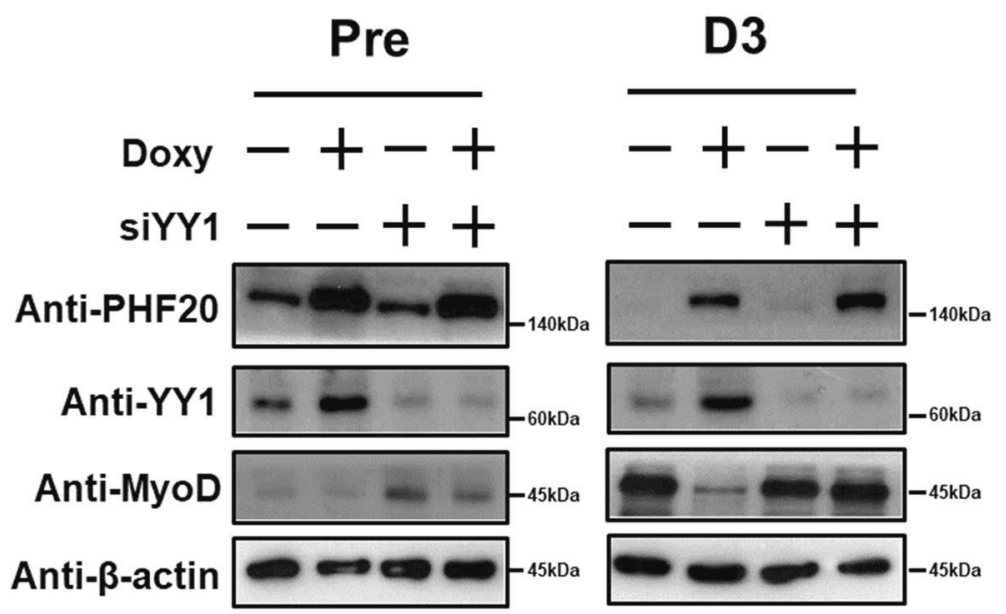

B

D1
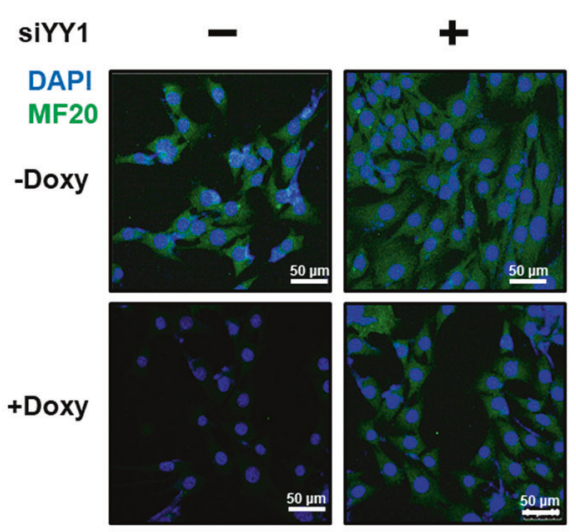

C

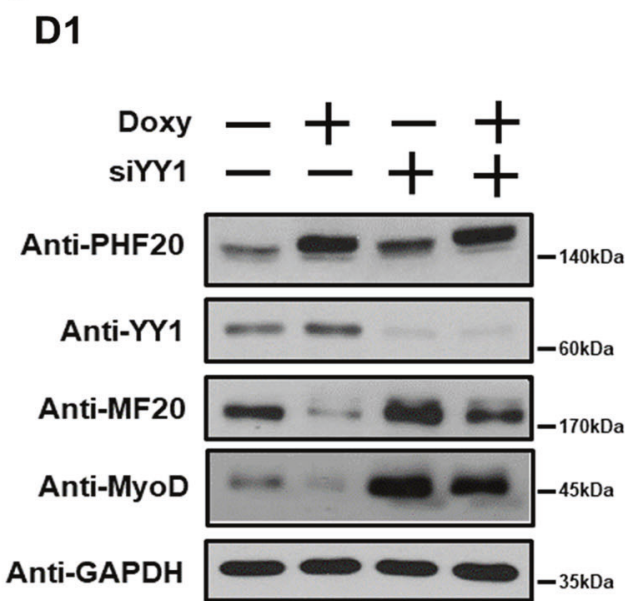

confirmed using immunohistochemistry (Fig. 5i, j). Another interesting observation was that there was little adipose tissue between the muscles of WT mice, whereas adipose

tissues were found between the muscles of PHF20-TG mice, detected by perilipin staining (Fig. 5k). Thus, PHF20TG mice did not have the same shapes and lengths of 
Fig. 4 PHF20 inhibits myogenic differentiation marker (MyoD) expression via the YY1 promoter. a $\mathrm{C}_{2} \mathrm{C}_{12}$ cells were differentiated with induction medium. At different time points (Pre and D3), $\mathrm{C}_{2} \mathrm{C}_{12}$ cells were transfected with control siRNA or siYY1 without/with doxycycline $(100 \mathrm{ng} / \mathrm{mL})$. Cell lysates were analyzed by immunoblotting with the corresponding antibodies. Results are representative of three independent experiments. $\mathbf{b} \mathrm{C}_{2} \mathrm{C}_{12}$ cells were differentiated on day 1 (D1). At that time, $\mathrm{C}_{2} \mathrm{C}_{12}$ cells were transfected with control siRNA or siYY1 without/with doxycycline $(100 \mathrm{ng} / \mathrm{mL}) . \mathrm{C}_{2} \mathrm{C}_{12}$ cells were processed by immunofluorescence with an anti-MF20 antibody (green). Nuclei were counterstained with DAPI (blue). The samples were analyzed using confocal microscopy. Scale bars, $50 \mu \mathrm{m} . \mathrm{c}_{2} \mathrm{C}_{12}$ cells were differentiated on day 1 (D1). At that time, $\mathrm{C}_{2} \mathrm{C}_{12}$ cells were transfected with control siRNA or siYY1 without/with doxycycline (100 ng/mL). Expression levels of PHF20, YY1, MF20, and MyoD were determined using immunoblotting with corresponding antibodies. Results are expressed as the mean \pm SEM of three independent experiments $(* * p<0.01 ; * p<0.05)$, using one-way ANOVA with Tukey's correction for multiple comparisons.

muscle fibers, meaning that muscles did not develop normally in these mice. Taken together, the results showed that PHF20 had a negative effect on muscle differentiation, both in vitro and in vivo.

\section{Regeneration of CTX-induced muscle damage is delayed in PHF20-TG mice}

To further investigate the negative roles of PHF20 in myogenic differentiation, an experiment for muscle regeneration after injury was performed in PHF20-TG mice. When cardiotoxin (CTX) is injected into the mouse TA muscle, the cells are damaged and destroyed, and over time, regeneration occurs and the muscles are restored. Based on this phenomenon, CTX was used in muscle regeneration research [13]. To investigate the degree of muscle regeneration in PHF20-TG mice, CTX was injected into TA muscle and TA muscle was isolated at 0,5 , and 10 days to check the degree of muscle regeneration through $\mathrm{H} \& \mathrm{E}$ staining and immunofluorescence, respectively. Interestingly, the regenerative capability of TA muscle was dramatically reduced in PHF20-TG mice compared with that in WT mice (Fig. 6a, b). As shown by reduction in the size of myofibers in PHF20-TG mice compared with WT after 5 and 10 days of injury, PHF20 inhibits skeletal muscle regeneration in vivo. Next, the ability of PHF20 to regulate the myogenic differentiation of muscle satellite cell was further evaluated. Satellite cells were isolated from the skeletal muscle of WT and PHF20-TG mice. After 3 days in DM, PHF20-TG satellite cells showed delay in myotube formation, as indicated by a lower differentiation index (MF20 staining) compared with WT satellite cells (Fig. 6c). In addition, to compare the expression level of PHF20 and its downstream YY1 in satellite cells after injury-induced regeneration, noninjury satellite cells and regenerated satellite cells were isolated from WT and PHF20-TG mice.
After regeneration, PHF20 and YY1 protein levels were reduced in WT satellite cells than noninjury satellite cells, and MyoD expression was increased. On the other hand, PHF20-TG satellite cells had higher expression levels of PHF20 and YY1 than noninjury cells. Interestingly, in the satellite cells of PHF20-TG in which regeneration occurred, the level of MyoD was significantly reduced (Fig. 6d, left panel, last lane). These results provide the clear evidence that muscle regeneration after injury was delayed in PHF20TG mice.

\section{Discussion}

PHF20 has been previously described as a unique candidate for cancer-specific immunotherapy [19, 20]. PHF20 has also been shown to be essential for the normal development of embryos [3]. PHF20 performs two functions: (1) PHF20 controls signal transduction events for transcription factors [14], and (2) PHF20 acts as a transcription factor that regulates protein expression levels [2, 21-23]. Although PHF20 has been extensively studied, its role and function in metabolism have not yet been reported.

Recent studies have shown that $\mathrm{TNF} \alpha / \mathrm{NF}-\kappa \mathrm{B}$ signaling regulates myogenic differentiation by modulating $\mathrm{MyoD}$ stability, which plays a negative role in myogenesis [24]. In this regard, our results showed that PHF20 expression was positively correlated with YY1 expression in myogenic differentiation (Fig. 1a-c). Consistent with the previous YY1 studies [16, 24], which reported that YY1 negatively affected myogenic terminal differentiation, YY1 expression was decreased during myogenic differentiation (Fig. 1d-f). The positive correlation between PHF20 and YY1 expression indicated that PHF20, together with YY1, may play major negative roles in myogenesis. Interestingly, the protein form of PHF20 was barely detected at differentiation days 2 and 3 (Fig. 1b), suggesting that PHF20 may be involved in an early stage of myogenic differentiation. Overexpression of PHF20 further revealed that forced expression of PHF20, mediated by doxycycline, resulted in stable expression of YY1 and a concomitant delay of MyoD expression during myogenic differentiation (Fig. 2a). Similar changes of mRNA expression were also observed (Fig. 2b). In addition, the events of terminal differentiation (e.g., myotube formation) were also delayed, which was monitored by MHC immunostaining (Fig. 2c). These results were further confirmed in $\mathrm{C}_{2} \mathrm{C}_{12}$ cells with PHF20 depletion, by shRNA-knockdown (Fig. 2d, e), clearly demonstrating that PHF20 played a negative role in myogenic differentiation together with YY1. Similar to our results, Msh homeobox1 has been reported to cooperate with PKCrelated kinase 1 to prevent differentiation of myogenic precursor cells [25]. Contrary to our results, Kim and co- 

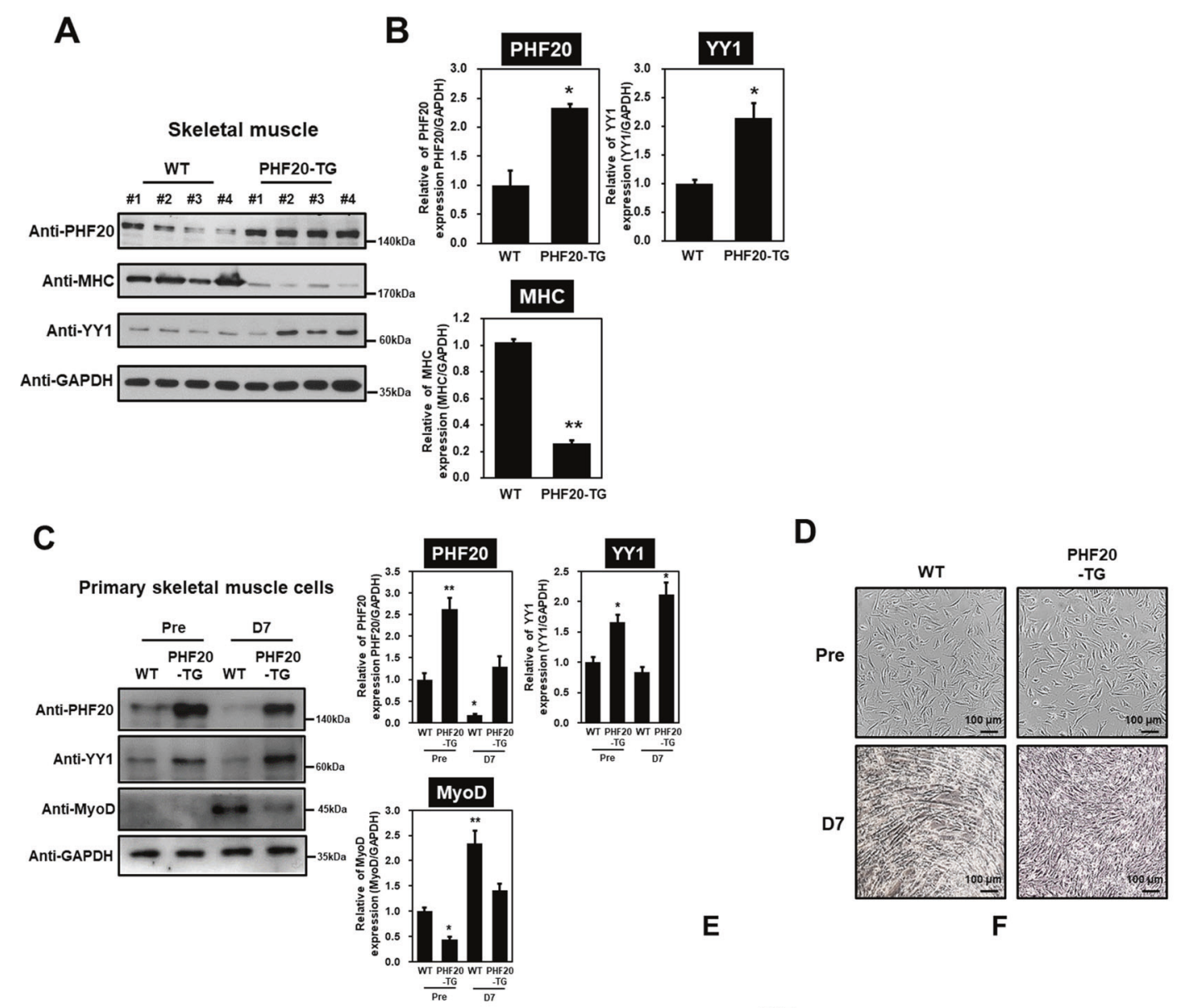

G

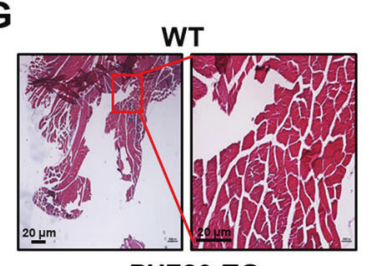

PHF20-TG

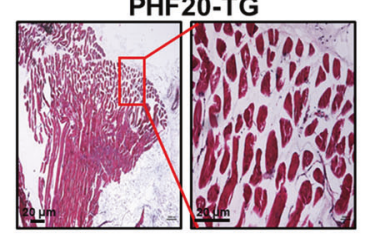

I

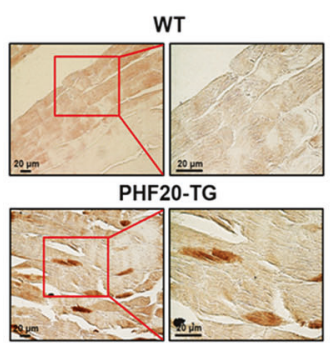

H
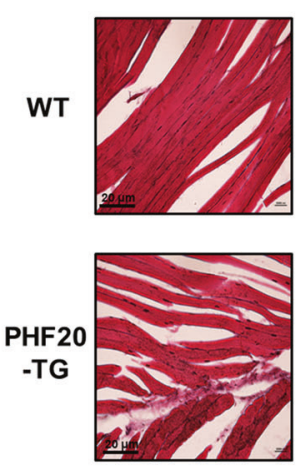

J
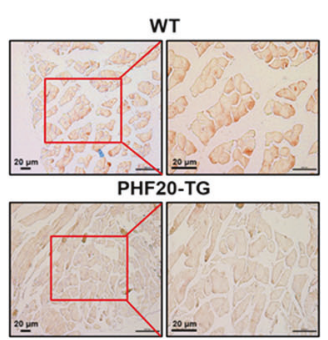

WT
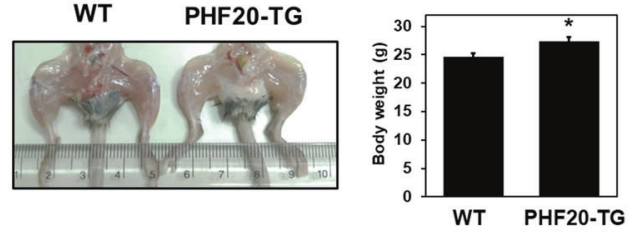

K

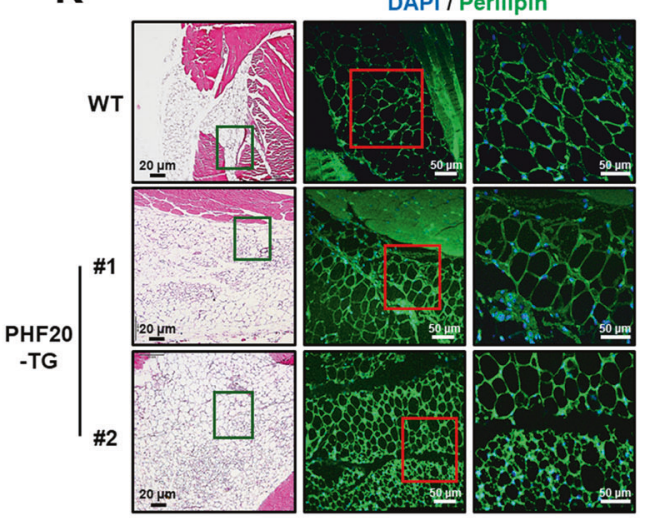

workers reported that the $\beta 2$-adrenergic receptor agonist, formoterol, blocked myogenesis by blocking the PI3K-PKB pathway [26]. However, since PKB activation has not yet been reported to increase the protein level of PHF20, further studies of possible direct correlations are needed. Podocan, a small leucine-rich repeat protein, has 
4 Fig. 5 Whole calf muscle and skeletal muscle shape and morphology differ between wild-type (WT) and PHF20 transgenic (TG) mice. a Lysates from skeletal muscle tissues derived from 5month-old male WT and PHF20-TG mice $(n=4)$. Expression levels of PHF20, YY1, and MHC were determined using western blot analysis with the corresponding antibodies. Results are representative of three independent experiments. b Relative and statistical differences of PHF20, YY1, and MHC in skeletal muscle were plotted. Data are expressed as the mean \pm SEM $(* p<0.05 ; * *<0.01)$ ' using Student's $t$ test for paired samples. c Lysates from primary skeletal myoblast cells derived from 3-month-old male WT and PHF20-TG mice $(n=6)$. Expression levels of PHF20, YY1, and MyoD were analyzed by immunoblotting with the corresponding antibodies. Results are representative of three independent experiments. Data are expressed as the mean $\pm \operatorname{SEM}(* * p<0.01 ; * p<0.05)$ ' using one-way ANOVA with Tukey's correction for multiple comparisons. d Primary myoblasts images of WT and PHF20-TG mice were differentiated for 7 days showing a delayed myotube formation in PHF20-TG primary cells. e The appearance of muscle and calves of WT and PHF20-TG mice. f Body weight of 5-month-old male WT and PHF20-TG mice. g Cross sections of the gastrocnemius from 5-month-old male WT or PHF20TG mice stained with hematoxylin and eosin (h, e). Scale bars, $20 \mu \mathrm{m}$. h Longitudinal sections from 5-month-old male WT or PHF20-TG mice stained with H\&E. Scale bars, $20 \mu \mathrm{m}$. i Cross section of the gastrocnemius from 5-month-old male WT or PHF20-TG mice stained with anti-MHC using immunohistochemistry. Scale bars, $20 \mu \mathrm{m}$. j Longitudinal sections of a skeletal muscle from 5-month-old male WT or PHF20-TG mice stained with anti-MHC using immunohistochemistry. Scale bars, $20 \mu \mathrm{m}$. k Cross section of fat between the gastrocnemius from 5-month-old male WT or PHF20-TG mice stained with H\&E. Scale bars, $20 \mu \mathrm{m}$, or with anti-perilipin antibody (green). Nuclei were counterstained with DA (blue). The samples were analyzed using confocal microscopy. Scale bars, $50 \mu \mathrm{m}$.

also been shown to regulate the differentiation of $\mathrm{C}_{2} \mathrm{C}_{12}$ myoblasts [27]. Notably, podocan function is opposed to PHF20 function. Overexpression of podocan enhances myogenic differentiation by activating $\mathrm{Wnt} / \beta$-catenin signaling. Therefore, it is possible that PHF20 and podocan negatively regulate each other to control myogenic differentiation, suggesting that myogenic differentiation is more complex, and involves many proteins.

As a transcription factor, PHF20 has been shown to regulate the transcription of several genes [2, 21-23], including p53, which is central regulator for cell death, cell survival, cell growth arrest, and proliferation [1]. When there is no growth factor in cells, PHF20 binds to the promoter of p53 and initiates transcription to cause cell death and cell growth arrest [1]. Conversely, stimulation by a growth factor such as glucose induces PHF20 to be phosphorylated by PKB and released from the promoter of $\mathrm{p} 53$, leading to cell survival and proliferation. Another transcriptional target of PHF20 is Oct4, which causes the reprogramming of induced pluripotent stem cells [2]. In breast cancer cells, it also activates Oct4 transcription and inhibits tumorigenesis [23]. In our studies, PHF20 directly binds and regulates YY1 promoter to control myogenic differentiation (Fig. 3). Consequently, YY1 inhibits the expression of genes involved in differentiation. When differentiation occurs, PHF20 is released from the YY1 promoter, leading to inhibition of YY1 expression by decreasing the levels of histone $\mathrm{H} 4$ acetylation (Supplementary Fig. S1). Depletion of YY1 in the myogenic differentiation revealed that PHF20 effects on MyoD expression (Fig. 4a) and myotube formation (Fig. 4b) was dependent on YY1 expression. Notably, differentiation was restored, judged by MyoD expression, when YY1 was depleted, despite overexpression of PHF20 (Fig. 4a, right panel, last lane), suggesting that PHF20 indirectly modulated differentiation regulatory factors, such as MyoD, through YY1. In the similar context, Zhou et al. have reported that large intergenic noncoding RNA for YY1 (linc-YY1) bind directly to YY1 and has a positive role in muscle differentiation and regeneration by evicting YY1/ polycomb repressive complex 2 complex [7]. Surprisingly, during the myogenic differentiation, linc-YY1 expression was suppressed in PHF20-overexpressed $\mathrm{C}_{2} \mathrm{C}_{12}$ cells compared with control cells (Supplementary Fig. S2A). Furthermore, when PHF20 was depleted by siRNA, the expression of linc-YY1 was remarkably elevated in premyocyte as well as differentiated day-1 cells (Supplementary Fig. S2B). The similar results were also observed in PHF20-TG mice (Supplementary Fig. S3), indicating that linc-YY1 expression is inversely correlated to PHF20 expression. Therefore, it will be interesting to see how PHF20 can affect linc-YY1 transcription in the molecular levels in vivo.

To further evaluate the in vivo function of PHF20, PHF20-TG mice has been generated. Characterization of PHF20-TG mice revealed that YY1 expression was upregulated in skeletal muscle with notable reduction of MHC expression (Fig. 5a, b). In addition, the ability of myogenic differentiation in primary muscle cells from PHF20-TG mice was also inhibited, judged by low expression of $\mathrm{MyoD}$ (Fig. 5c) and myotube formation (Fig. 5d). Consistent with the phenotype in PHF20-KO mice (a reduction of fat storage; [3]), the muscle color of PHF20-TG mice was pale (Fig. 5e), indicating that fat accumulation occurred in these mice. It was assumed that the muscles appeared pale due to high accumulation of fat. Weight gain phenotype of PHF20TG mice further support these observations (Fig. 5f). Furthermore, dissection of muscles confirmed that fat indeed accumulated between the surface of muscles and the spaces between muscle fibers (Fig. $5 \mathrm{~g}, \mathrm{~h}, \mathrm{k}$ ). In this regard, it has been reported that muscle fibers were formed in short and thick irregular shapes rather than in long and straight shapes, when muscle differentiation was interrupted by any stimulus or the differentiation gene did not function properly [8]. PHF20-TG mice displayed the small and irregularsized muscle fibers. A large amount of empty space between each muscle fiber was also found in PHF20-TG mice (Fig. 5g, h). In addition, PHF20 was able to regulate muscle 


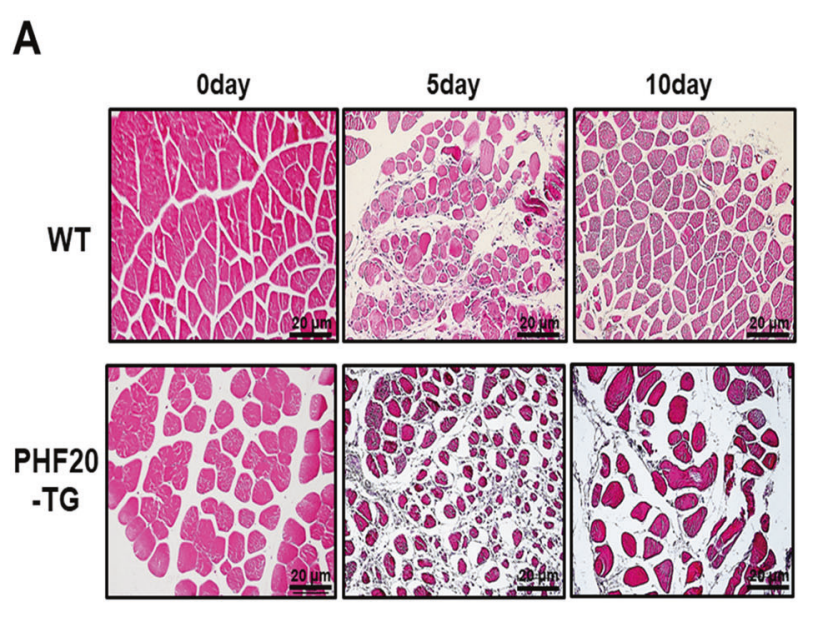

\section{B}
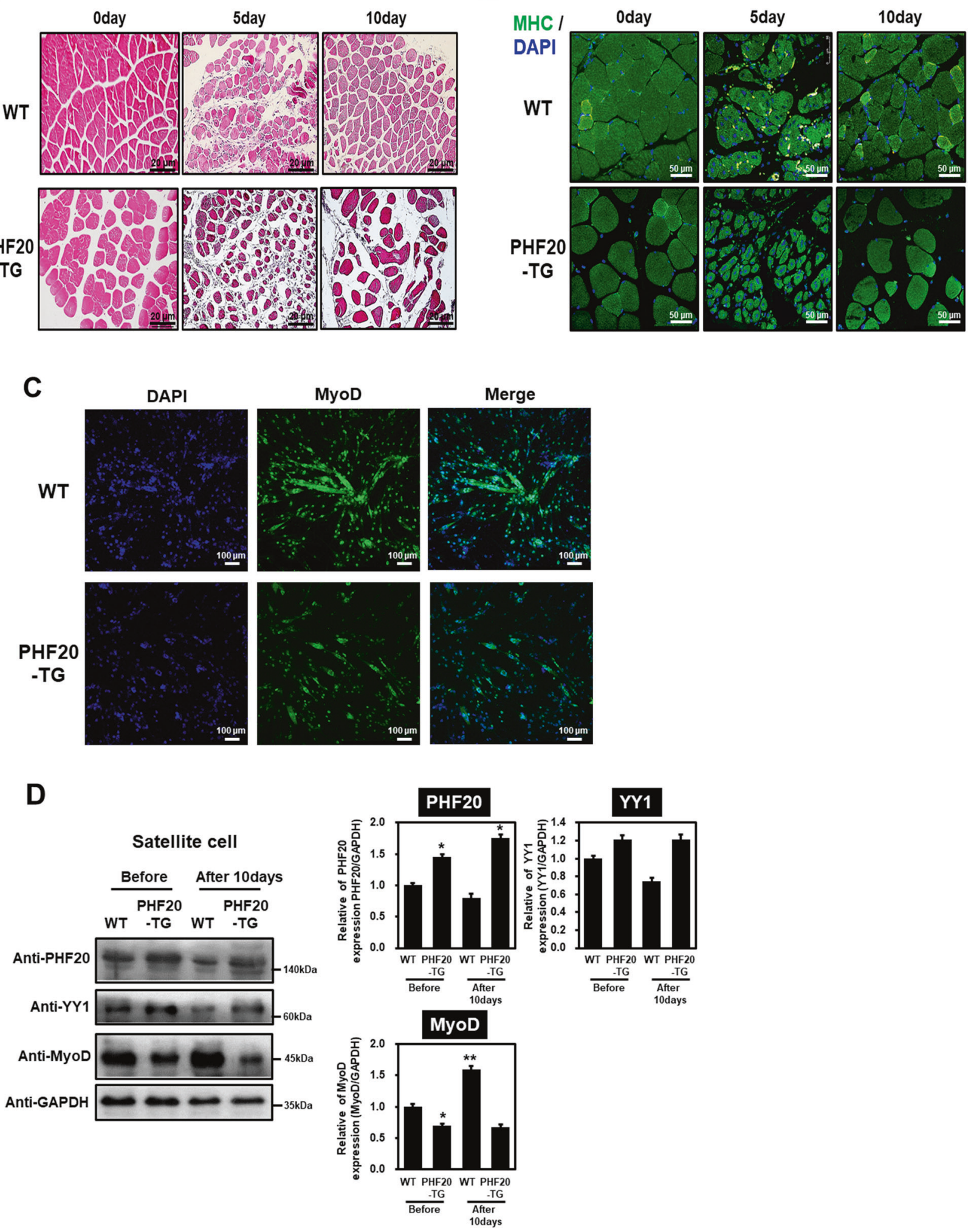

regeneration since myoblast differentiation is involved in the regeneration process after injury. As shown in Fig. 6a, b, regeneration in PHF20-TG muscle was delayed compared with WT muscle when CTX-induced damage was introduced in TA muscle. Similar to these observation, PHF20 also can inhibit the myogenic differentiation of muscle 
Fig. 6 Overexpression of PHF20 delays muscle regeneration after CTX-injury. a H\&E stained sections of TA muscles from WT and PHF20-TG mice showing a delayed regeneration of injured TA muscle in PHF20-TG mice compared with that in WT littermates at 5 and 10 days $(n=4)$ after CTX injection. Scale bar, $20 \mu \mathrm{m}$. b Overlaid photomicrographs of TA muscle sections of WT and PHF20-TG mice 0,5 , and 10 days $(n=4)$ post injury after immunostaining for MHC (green). Nuclei were counterstained with DAPI (blue). Scale bar, $50 \mu \mathrm{m}$. c Satellite cells derived from 3-month-old male WT and PHF20-TG mice $(n=5)$ were differentiated on day 3 (D3). Satellite cells were processed by immunofluorescence with an anti-MyoD antibody (green). Nuclei were counterstained with DAPI (blue). The samples were analyzed using confocal microscopy. Scale bars, $100 \mu \mathrm{m}$. d Immunoblots analysis demonstrate the PHF20, YY1, MyoD, and GADPH protein levels in differentiated for 10 days satellite cell from noninjury TA muscle or in day 10 post-injury TA muscle of WT and PHF20-TG mice $(n=5)$. Relative and statistical differences of PHF20, YY1, and MyoD in satellite cells were plotted. Data are expressed as the mean \pm SEM $(* p<0.05 ; * * p<0.01)$, using one-way ANOVA with Tukey's correction for multiple comparisons.

satellite cells in before/after injury-induced regeneration process (Fig. 6c, d), suggesting that PHF20 is also essential for early stage of skeletal muscle regeneration.

Taken together, this reports provided a clear in vivo evidence that PHF20 plays a negative role in myogenesis and damage-induced muscle regeneration via YY1. The proposed mechanism of PHF20 action on myogenic differentiation is the following: (1) in premyoblasts, PHF20 directly binds to the promoter of YY1 and activates YY1 transcription to inactivate the genes involved in differentiation. (2) During differentiation, PHF20 is detached from the YY1 promoter, which inhibits YY1 transcription, thereby activating differentiation genes, including MyoD and myogenin (Fig. 7). Several studies have reported that type 2 diabetes occurs due to a decrease in the amount of muscle, so the importance of muscle is now becoming clear. In this regards, it is possible that PHF20 may be a useful target in treating obesity and diabetic patients with

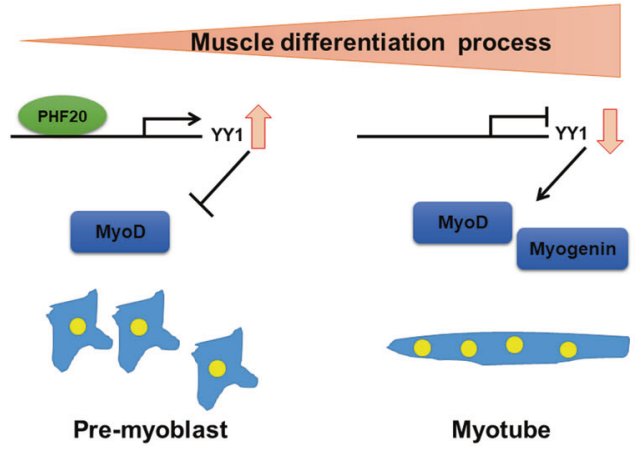

Fig. 7 Schematic illustration of the working model for the role of PHF20 during myogenesis. During muscle differentiation, PHF20 directly regulates the expression of YY1 and ultimately affects differentiation. At the myoblast stage, PHF20 binds to the YY1 promoter and enhances YY1 expression. When differentiation begins, PHF20 is released from the promoter of YY1 and expression of YY1 is reduced. myopenia or muscular dystrophy. In addition, PHF20 may also be effective in the prevention and treatment of muscle loss during aging.

Acknowledgements This work was financially supported by research fund of Chungnam National University (Jongsun Park) and the National Research Foundation of Korea (NRF) grant funded by the Korea Government (MEST) (NRF-2014R1A1A3050752, NRF2015R1A2A2 A01003597). The English in this document has been checked by at least two professional editors, both native speakers of English. For a certificate, please see: http://www.textcheck.com/ certificate/KMV0G8.

\section{Compliance with ethical standards}

Conflict of interest The authors declare that they have no conflict of interest.

Publisher's note Springer Nature remains neutral with regard to jurisdictional claims in published maps and institutional affiliations.

\section{References}

1. Cui G, Park S, Badeaux AI, Kim D, Lee J, Thompson JR, et al. PHF20 is an effector protein of p53 double lysine methylation that stabilizes and activates p53. Nat Struct Mol Biol. 2012;19:916-24.

2. Zhao W, Li Q, Ayers S, Gu Y, Shi Z, Zhu Q, et al. Jmjd3 inhibits reprogramming by upregulating expression of INK4a/Arf and targeting PHF20 for ubiquitination. Cell. 2013;152:1037-50.

3. Badeaux AI, Yang Y, Cardenas K, Vemulapalli V, Chen K, Kusewitt D, et al. Loss of the methyl lysine effector protein PHF20 impacts the expression of genes regulated by the lysine acetyltransferase MOF. J Biol Chem. 2012;287:429-37.

4. Sanchez AM, Candau RB, Bernardi H. FoxO transcription factors: their roles in the maintenance of skeletal muscle homeostasis. Cell Mol Life Sci. 2014;71:1657-71.

5. Wu AL, Kim JH, Zhang C, Unterman TG, Chen J. Forkhead box protein $\mathrm{O} 1$ negatively regulates skeletal myocyte differentiation through degradation of mammalian target of rapamycin pathway components. Endocrinology. 2008;149:1407-14.

6. Knight JD, Kothary R. The myogenic kinome: protein kinases critical to mammalian skeletal myogenesis. Skelet Muscle. 2011;1:29.

7. Zhou L, Sun K, Zhao Y, Zhang S, Wang X, Li Y, et al. Linc-YY1 promotes myogenic differentiation and muscle regeneration through an interaction with the transcription factor YY1. Nat Commun. 2015;6:10026.

8. Blattler SM, Cunningham JT, Verdeguer F, Chim H, Haas W, Liu $\mathrm{H}$, et al. Yin Yang 1 deficiency in skeletal muscle protects against rapamycin-induced diabetic-like symptoms through activation of insulin/IGF signaling. Cell Metab. 2012;15:505-17.

9. Cunningham JT, Rodgers JT, Arlow DH, Vazquez F, Mootha VK, Puigserver P. mTOR controls mitochondrial oxidative function through a YY1-PGC-1alpha transcriptional complex. Nature. 2007; 450:736-40.

10. Ji K, Zheng J, Lv J, Xu J, Ji X, Luo YB, et al. Skeletal muscle increases FGF21 expression in mitochondrial disorders to compensate for energy metabolic insufficiency by activating the mTOR-YY1-PGClalpha pathway. Free Radic Biol Med. 2015;84:161-70.

11. Tran Q, Jung JH, Park J, Lee H, Hong Y, Cho H, et al. S6 kinase 1 plays a key role in mitochondrial morphology and cellular energy flow. Cell Signal. 2018;48:13-24. 
12. Jin M, Hong $\mathrm{Y}$, Lee $\mathrm{H}$, Tran $\mathrm{Q}$, Cho $\mathrm{H}$, Kim M, et al. 1,2Dichloropropane (1,2-DCP)-induced angiogenesis in dermatitis. Toxicol Res. 2019;35:361-9.

13. Garry GA, Antony ML, Garry DJ. Cardiotoxin Induced Injury and skeletal muscle regeneration. Methods Mol Biol. 2016;1460:61-71.

14. Zhang T, Park KA, Li Y, Byun HS, Jeon J, Lee Y, et al. PHF20 regulates NF-kappaB signalling by disrupting recruitment of PP2A to p65. Nat Commun. 2013;4:2062.

15. Lee TC, Shi Y, Schwartz RJ. Displacement of BrdUrd-induced YY1 by serum response factor activates skeletal alpha-actin transcription in embryonic myoblasts. Proc Natl Acad Sci USA. 1992;89:9814-8.

16. Vincent CK, Gualberto A, Patel CV, Walsh K. Different regulatory sequences control creatine kinase-M gene expression in directly injected skeletal and cardiac muscle. Mol Cell Biol. 1993;13:1264-72.

17. Klein BJ, Wang X, Cui G, Yuan C, Botuyan MV, Lin K, et al. PHF20 readers link methylation of histone H3K4 and p53 with H4K16 acetylation. Cell Rep. 2016;17:1158-70.

18. Chinnappan D, Xiao D, Ratnasari A, Andry C, King TC, Weber HC. Transcription factor YY1 expression in human gastrointestinal cancer cells. Int J Oncol. 2009;34:1417-23.

19. Fischer U, Struss AK, Hemmer D, Pallasch CP, Steudel WI, Meese E. Glioma-expressed antigen 2 (GLEA2): a novel protein that can elicit immune responses in glioblastoma patients and some controls. Clin Exp Immunol. 2001;126:206-13.

20. Pallasch CP, Struss AK, Munnia A, Konig J, Steudel WI, Fischer $\mathrm{U}$, et al. Autoantibodies against GLEA2 and PHF3 in glioblastoma: tumor-associated autoantibodies correlated with prolonged survival. Int J Cancer. 2005;117:456-9.
21. Yang JW, Jeong BC, Park J, Koh JT. PHF20 positively regulates osteoblast differentiation via increasing the expression and activation of Runx2 with enrichment of H3K4me3. Sci Rep. 2017;7:8060.

22. Zhang C, Gao W, Dong S, Chen G, Han R, Wen S, et al. [The expression and clinical significance of Bax and PHF20 in laryngeal squamous cell carcinoma]. Lin Chung Er Bi Yan Hou Tou Jing Wai Ke Za Zhi = J Clin Otorhinolaryngol, Head, Neck Surg. 2015;29:1701-5.

23. Xun J, Wang D, Shen L, Gong J, Gao R, Du L, et al. JMJD3 suppresses stem cell-like characteristics in breast cancer cells by downregulation of Oct 4 independently of its demethylase activity. Oncotarget. 2017;8:21918-29.

24. Langen RC, Van Der Velden JL, Schols AM, Kelders MC, Wouters EF, Janssen-Heininger YM. Tumor necrosis factor-alpha inhibits myogenic differentiation through MyoD protein destabilization. FASEB J: Off Publ Federation Am Societies Exp Biol. 2004;18:227-37.

25. Zhu X, Li M, Jia X, Hou W, Yang J, Zhao H, et al. The homeoprotein Msx1 cooperates with Pkn1 to prevent terminal differentiation in myogenic precursor cells. Biochimie. 2019;162:55-65.

26. Kim SH, Yi SJ, Lee H, Kim JH, Oh MJ, Song EJ, et al. beta2Adrenergic receptor (beta2-AR) agonist formoterol suppresses differentiation of L6 myogenic cells by blocking PI3K-AKT pathway. Anim cells Syst. 2019;23:18-25.

27. Liu D, Li S, Cui Y, Tong H, Li S, Yan Y. Podocan affects C2C12 myogenic differentiation by enhancing Wnt/beta-catenin signaling. J Cell Physiol. 2019;234:11130-9. 